I. Rytöluoto, M. Ritamäki, K. Lahti, M. Paajanen, M. Karttunen, G. C. Montanari, P. Seri and H. Naderiallaf "Compounding, Structure and Dielectric Properties of Silica-BOPP Nanocomposite Films, " in 2018 IEEE 2nd International Conference on Dielectrics (ICD), Budapest, 2018, pp. 1-4.

The published version is available online at:

https://doi.org/ 10.1109/ICD.2018.8514775

(C) 2018 IEEE. Personal use of this material is permitted. Permission from IEEE must be obtained for all other uses, in any current or future media, including reprinting/republishing this material for advertising or promotional purposes, creating new collective works, for resale or redistribution to servers or lists, or reuse of any copyrighted component of this work in other works. 


\title{
Compounding, Structure and Dielectric Properties of Silica-BOPP Nanocomposite Films
}

\author{
Ilkka Rytöluoto, Mikael Ritamäki, Kari Lahti \\ Laboratory of Electrical Energy Engineering (EEE) \\ Tampere University of Technology (TUT) \\ Tampere, Finland \\ ilkka.rytoluoto@tut.fi
}

\author{
Mika Paajanen, Mikko Karttunen \\ VTT Technical Research Centre of Finland \\ Tampere, Finland
}

\author{
Gian Carlo Montanari, Paolo Seri, Hadi Naderiallaf \\ Electrical, Electronic and Information Engineering "Guglielmo Marconi” Dept. (DEI) \\ University of Bologna \\ Bologna, Italy
}

\begin{abstract}
This study presents preliminary results on the morphology, structure and dielectric properties of laboratory scale cast and biaxially oriented silica-PP films, currently being developed in the European project GRIDABLE. Space charge measurements on the thick cast films indicated a reduction of positive space charge and faster depolarization characteristics upon inclusion of nano-silica, being indicative of reduction of trap depth and density distribution in comparison to neat PP. Likewise, for the biaxially oriented silica-PP thin films, increased amount of shallow traps and a reduction of concentration of traps in the deep trap range $(\sim 1 \mathrm{eV})$ were observed. Nano-silica was also found to reduce the dielectric loss at high temperatures in the frequency range appropriate to use. Such dielectric property modifications can potentially lead to benefits for insulation reliability and lifetime, especially at higher temperatures. A two-step compounding via a master batch approach was also found to be a viable option for the up-scaling of nanocomposite production.
\end{abstract}

Keywords-polypropylene; silica; nanocomposite; thin film processing; space charge; trapping; permittivity; dielectric loss

\section{INTRODUCTION}

Dielectric polymer nanocomposites (PNCs) are among the most interesting material candidates to overcome challenges of the currently employed polymeric HVDC insulations [1, 2]. Biaxially oriented polypropylene (BOPP) nanocomposites incorporating low amounts of hydrophobic fumed silica nanoparticles have previously been shown to exhibit promising short- and medium-term dielectric properties such as improved breakdown strength [3], increased partial discharge endurance [4], reduced space charge accumulation [5] and no pronounced differences in medium-term DC electro-thermal ageing behavior [6] in comparison to neat BOPP. Such dielectric property improvements are highly desired for applications in metallized film capacitors and DC cables. For instance, space charge accumulation in conventional polymers under high DC electric field can degrade severely the insulation reliability and reduce lifetime of DC cable systems and film capacitors [7]-[9]. Considering that both cables and capacitors are critical components in HVDC transmission and distribution systems, as well as in voltage source converters (VSC) used for gridintefacing of renewables, increasing their life and/or reliability is of high interest. Another issue for metallized film capacitors is the insufficient electrostatic energy density and operation temperature of the current state-of-the-art BOPP films [10] which inevitably leads to high capacitor volume and mass [11].

Next-generation PP-silica nanocomposites to be utilized in DC cables and capacitors are currently being developed in the European project GRIDABLE. It is known from our previous studies that optimized nanoparticle surface functionalization, pre-mixing, compounding and film manufacturing practices under clean processing conditions are pivotal in achieving highdielectric-performance biaxially oriented thin films $[3,12,13]$. Thus, as a preparatory stage for a forthcoming industrial-scale material production trial, silica-BOPP films manufactured in laboratory scale are currently being studied for establishing optimized material formulations and processing procedures. Preliminary results on their morphology, structure and dielectric properties, especially charge trapping and transport properties, are presented herein.

\section{EXPERIMENTAL}

\section{A. Materials and film manufacturing}

Compounds comprising of a capacitor-grade isotactic polypropylene (PP) homopolymer matrix and 0-4.5 wt- $\%$ of hydrophobic fumed silica nanoparticles were manufactured using a KraussMaffei Berstorff ZE $25 \times 49$ D UTX compounder (screw speed $150 \mathrm{rpm}$, output $7 \mathrm{~kg} / \mathrm{h}$; nitrogen gas and a screen pack were used). The compounded strands were cooled in water bath, pelletized and then extruded into $\sim 400 \mu \mathrm{m}$ thick cast films using a Brabender Plasticorder single screw extruder inside a mobile soft-walled clean room. Two $1.0 \mathrm{wt}-\%$ silica compounds were also prepared by diluting $4.5 \mathrm{wt} \%$ silica-PP masterbatch either by compounding (Masterbatch 1) or during cast film extrusion (Masterbatch 2). Finally, $10 \mathrm{~cm} \times 10 \mathrm{~cm}$ cast film specimens were biaxially stretched into $\sim 10-12 \mu \mathrm{m}$ thin films inside a semi-clean room using a Brückner KARO IV laboratory stretcher (temperature $\sim 157^{\circ} \mathrm{C}$, biaxial stretch ratio $5.6 \times 5.6$ ). 


\section{B. Space charge measurements}

Space charge measurements were performed for the thick cast films by the pulsed electro-acoustic (PEA) method, utilizing 10 ns voltage pulses with amplitude of $300 \mathrm{~V}$. The acoustic signals were collected by a $9 \mu \mathrm{m}$ thick PVDF piezoelectric and amplified by an amplifier cascade. The thin film system amplifier had a $-3 \mathrm{~dB}$ bandwidth of $250 \mathrm{MHz}$. These features resulted in a spatial resolution of less than $10 \mu \mathrm{m}$. Calibration of the system was made after $10 \mathrm{~s}$ from the polarization of the sample (under low voltage and electric field). The samples were pre-conditioned at $60^{\circ} \mathrm{C}$ under vacuum for 72 hours. The PEA measurements were done at $60{ }^{\circ} \mathrm{C}$, in a controlled environment, with fields of $50 \mathrm{~V} / \mu \mathrm{m}$. Polarization (when voltage is applied) and depolarization (when voltage is removed and the sample is short-circuited) times were $10800 \mathrm{~s}$ and $3600 \mathrm{~s}$, respectively.

C. Dielectric spectroscopy, thermally stimulated current and isothermal charging-discharging current measurements

Dielectric spectroscopy, thermally stimulated depolarization current (TSDC) and isothermal charging/ discharging current (ICC/IDC) experiments were performed for the biaxially stretched films. $\mathrm{Ni}+\mathrm{Au}(10 \mathrm{~nm}+100 \mathrm{~nm})$ electrodes were deposited on thin film specimens using a custom-built e-beam evaporator (Instrumentti Mattila) inside a clean room facility. High vacuum (pressure $<1 \times 10^{-6}$ mbar) and low deposition rate $(0.05-0.20 \mathrm{~nm} / \mathrm{s})$ were maintained during the evaporation process to minimize thermal and radiative damaging of the sample film. The evaporated samples were short-circuited and stored in a vacuumed desiccator for several days prior to electrical measurements. Complex relative permittivity $\varepsilon_{r}{ }^{*}=\varepsilon_{r}+i \varepsilon_{r}$ ' was measured using a Novocontrol Alpha-A dielectric analyzer equipped with a Novocool temperature control system. For TSDC and ICC/IDC measurements, a high voltage DC source (Keithley 2290E-5) and an electrometer (Keithley 6517B) were utilized. An overload protection circuit along with a series resistor was used to protect the electrometer in case of a sample breakdown during high voltage application. Shielded sample cell equipped with a PT100 temperature sensor was used for all the measurements. Temperature control (accuracy of $\pm 0.1^{\circ} \mathrm{C}$ ) was realized using the Novocool system or a PID-controlled electrical heating element.

For the TSDC measurement the procedure was: (i) polarization at DC field $E_{p}=100 \mathrm{~V} / \mu \mathrm{m}$ for $40 \mathrm{~min}$ under isothermal conditions at $T_{p}=80^{\circ} \mathrm{C}$, (ii) rapid cooling to $T_{0}=$ $-50{ }^{\circ} \mathrm{C}$; hold isothermally for $5 \mathrm{~min}$, (iii) removal of the poling voltage and short-circuiting of the sample through the electrometer; hold isothermally for $3 \mathrm{~min}$, (iv) linear heating at

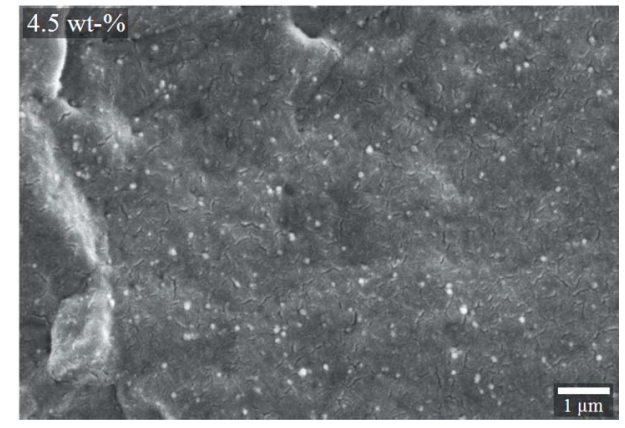

Fig. 1. Silica dispersion in a $4.5 \mathrm{wt}-\%$ silica-PP cast film.

$\beta=3.0^{\circ} \mathrm{C} / \mathrm{min}$ up to $T_{\max }=125^{\circ} \mathrm{C}$ while recording the depolarization current. For the ICC/IDC measurements the samples were held isothermally at $100{ }^{\circ} \mathrm{C}$ and charging current under DC electric field of $100 \mathrm{~V} / \mu \mathrm{m}$ was measured for $24 \mathrm{~h}$, followed by the measurement of discharge current for $6 \mathrm{~h}$.

\section{RESULTS AND DISCUSSION}

\section{A. Morphology and silica dispersion}

Fig. 1 shows a representative SEM image of a cryo-fractured $4.5 \mathrm{wt}-\%$ silica-PP cast film. In general, the nanocompounds were found to exhibit good silica dispersion and distribution in the PP matrix, although a few larger agglomerates and some impurities were observed.

\section{B. Space charge}

Fig. 2 presents space charge density profiles of selected silica-PP cast films along with an unfilled reference. Stored charge density at a specific field and depolarization time, $q(E, t)$, was derived from the space charge profile measurements as:

$$
q(E, t)=\frac{1}{L} \int_{0}^{L}\left|q_{p}(E, x, t) d x\right|
$$

where 0 and $L$ denote the electrodes positions and $q_{p}(E, x, t)$ is the space charge profile for a given poling field $E$. As shown in Fig. 2a, the unfilled PP reference accumulated a substantial amount of space charge over the polarization period at $60{ }^{\circ} \mathrm{C}$, leading to considerable electric field distortion within the bulk (maximum value was $\sim 1.4$ times the geometric (Laplacian) field). The situation was however greatly improved upon inclusion of nanosilica (Fig. 2b-c), with a general trend of reducing space charge with increasing silica content being evident (up to a nanofiller concentration limit which is generally near $5 \mathrm{wt}-\%)$. The silica-PP nanocompounds also showed faster depolarization characteristics in comparison to neat PP;
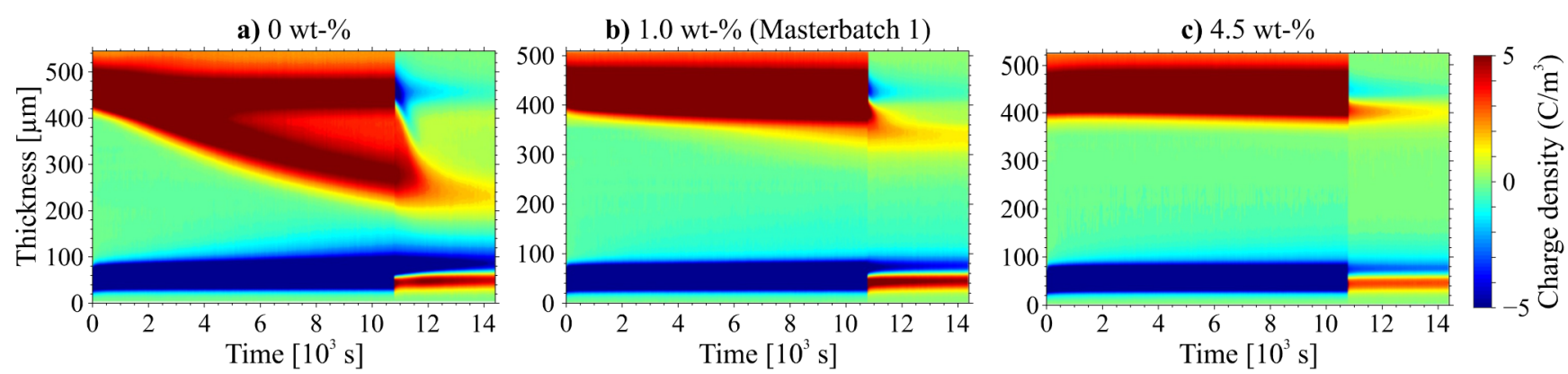

Fig. 2. Space charge density profiles of selected PP-silica cast films at $60{ }^{\circ} \mathrm{C}$ : a) unfilled reference, b) $1.0 \mathrm{wt}-\%$ silica-PP (diluted from 4.5 wt- $\%$ masterbatch by compounding, c) $4.5 \mathrm{wt}-\%$ silica-PP. Polarization field $(50 \mathrm{~V} / \mu \mathrm{m})$ was switched off at $10800 \mathrm{~s}$. Accumulation of positive space charge is observed in a). 
comparing the unfilled PP with the 4.5 wt-\% silica-PP, calculations on the charge depolarization trends indicated an increase in trap-controlled apparent mobility from $\sim 1.0 \times 10^{-14}$ $\mathrm{m}^{2} \mathrm{~V}^{-1} \mathrm{~s}^{-1}$ to $\sim 1.3 \times 10^{-12} \mathrm{~m}^{2} \mathrm{~V}^{-1} \mathrm{~s}^{-1}$ (for details, see [14]). Such results are indicative of reduction of trap depth and density distribution upon incorporation of nanosilica [5].

\section{Permittivity and dielectric loss}

Relative permittivity and dielectric loss characteristics of the biaxially stretched films as a function of temperature are presented in Fig. 3 along with a commercial capacitor BOPP reference from [15]. The measured $\varepsilon_{r}$ values were generally within the expected range for BOPP $[15,16]$, showing a slightly decreasing trend with increasing temperature from $-60{ }^{\circ} \mathrm{C}$ to 125 ${ }^{\circ} \mathrm{C}$. Although small differences in $\varepsilon_{r}$ were noticed between the studied materials, it is currently challenging to assess the role of silica alone, as such variations could also be attributed to film voids [3] and/or thickness measurement inaccuracy [17]. With increasing silica content, the dielectric loss spectra $\left(\tan \delta=\varepsilon_{r}{ }^{\prime} / \varepsilon_{r}\right)$ showed (i) a slight increase of $\tan \delta$ below the glass transition temperature $T_{g}$ (approx. $-5{ }^{\circ} \mathrm{C}[15]$ ), (ii) appearance of a new peak at $\sim 65{ }^{\circ} \mathrm{C}$ and (iii) reduction of dielectric loss at high temperatures. For the unfilled BOPP, the increase in $\tan \delta$ at high temperature is likely related to the release of trapped charge and increase of DC conductivity (the effect was much pronounced at low frequencies). This behavior was however mitigated for the silica-BOPP films. Overall, the $\tan \delta$ characteristics of the silicaBOPP films are very promising, especially at the expected operating temperatures, as maintaining a low dielectric loss level is a prerequisite in metallized film capacitor applications [10].

\section{D. $T S D C$}

Fig. 4 presents the TSDC spectra of selected compounds along with a commercial capacitor BOPP film from [15]. Based on the modified TSC theory, a numerical method proposed in [18] was used for direct determination of trap level vs. density distributions from the TSDC spectra, the results of which are exemplified in the inset of Fig. 4 for the shallow trap region. Upon heating from $-50{ }^{\circ} \mathrm{C}$, the first $\mathrm{TSC}$ peak was systematically observed around $T_{g}$ for all the films (trap depth $\sim 0.75 \mathrm{eV})$. For the silica compounds however, the TSC spectra above $T_{g}$ became more pronounced and complex, indicating higher presence of shallow traps with trap depths in the $0.75-0.9$ $\mathrm{eV}$ range in comparison to neat BOPP. In the high temperature

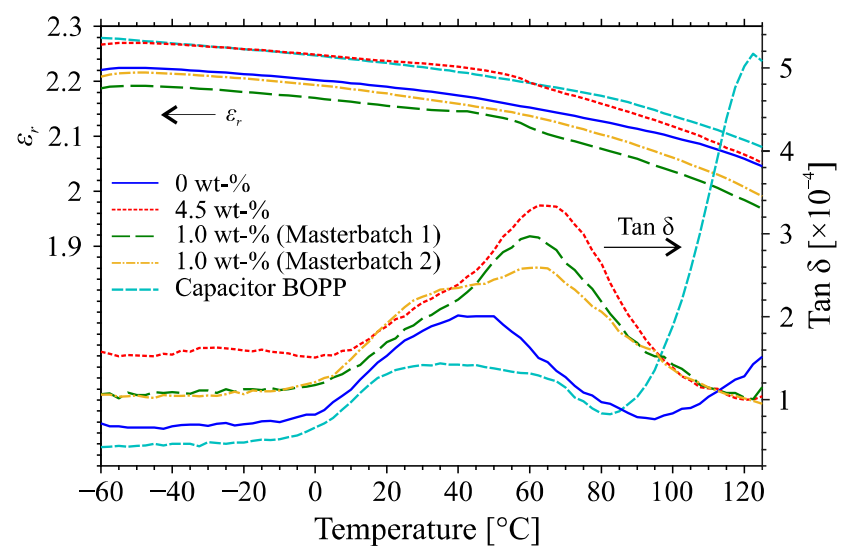

Fig. 3. Relative permittivity (real part) and dielectric loss $(\tan \delta)$ characteristics of the biaxially stretched thin films as a function of temperature $(1 \mathrm{kHz})$. region $\left(\sim 90{ }^{\circ} \mathrm{C}\right.$ and above), the unfilled BOPP films exhibited strong TSC peaks corresponding to charges released from deep traps (around 1.08-1.1 eV), often being anomalous in the sense that the current flow was in the same direction as the polarization current which is indicative of space charge and electrode effects $[15,19]$. For the silica-BOPP films however, the TSC in the deep trap region was greatly reduced, indicating that silica can effectively suppress deep traps. These results are consistent with the PEA space charge depolarization behavior measured from the thick cast films (Fig. 2) and confirm the modification of charge trapping properties also in silica-BOPP thin films.

\section{E. Isothermal charging-discharging current}

Isothermal charging current characteristics of selected biaxially stretched films at $100{ }^{\circ} \mathrm{C}$ are presented in Fig. 5a. A long-term power-law decay with no attainment of steady-state current during the $24 \mathrm{~h}$ polarization period was observed for all the films which is typical for BOPP $[15,20]$. A decrease of conductivity with increasing silica content was observed; comparing the unfilled reference with the $4.5 \mathrm{wt}-\%$ silica-BOPP film, the conductivity decreased from $6.9 \times 10^{-16} \mathrm{~S} / \mathrm{m}$ to $3.8 \times 10^{-}$ ${ }^{16} \mathrm{~S} / \mathrm{m}$. Fig. 5b shows the isothermal discharge current behavior measured after $24 \mathrm{~h}$ of poling, which, in agreement with the PEA depolarization characteristics of the corresponding thick cast films, indicate faster charge decay rate for the silica-BOPP films. Following the work of Simmons [21, 22], the isothermal discharge current density can be given as:

$$
J(t)=\frac{q l^{2} k T}{2 d t} f_{0}(E) N_{t}(E)
$$

where $q$ is the electronic charge, $l$ is the penetration depth of the injected electrons, $k$ is the Boltzmann's constant, $T$ is the absolute temperature, $d$ is the sample thickness, $f_{0}(E)$ is the initial occupancy of the traps (and approximately equal to 1 assuming that all the traps are occupied) and $N_{t}(E)$ is the energy distribution of the trap levels throughout the bandgap. The relation between trap level distribution $E_{t}(t)$ and time can be given as $E_{t}(t)=k T \ln (v t)$, where $v$ is the attempt-to-escape frequency $\left(\approx 10^{12} \mathrm{~s}^{-1}\right)[21,22]$. The inset in Fig. 5b shows the trap density vs. depth distributions derived from the ICC data. A considerable suppression of density of deep traps $(>1.1 \mathrm{eV})$ is observed for the silica-BOPP films, again being consistent with the PEA and TSDC measurements.

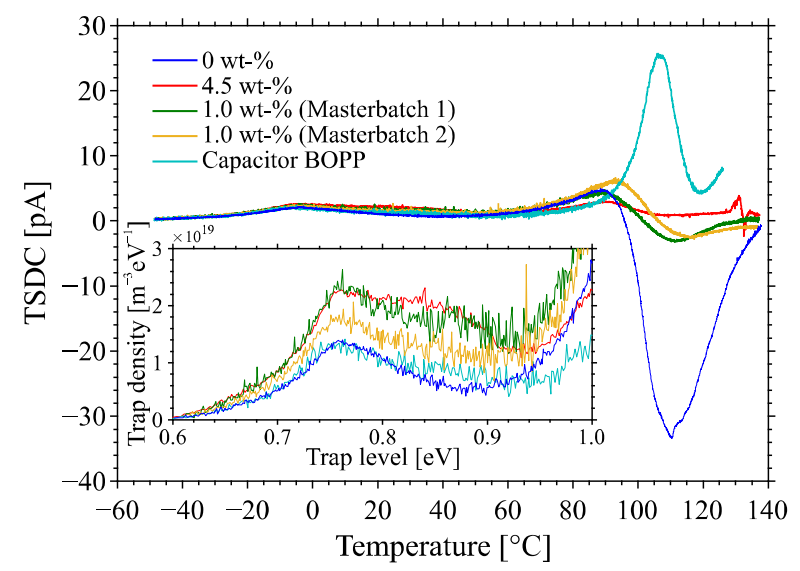

Fig. 4. Thermally stimulated depolarization current spectra of the biaxially stretched thin silica-PP films. The inset shows the trap level vs. density distributions in the shallow trap region (from -50 to $+85^{\circ} \mathrm{C}$ ). 

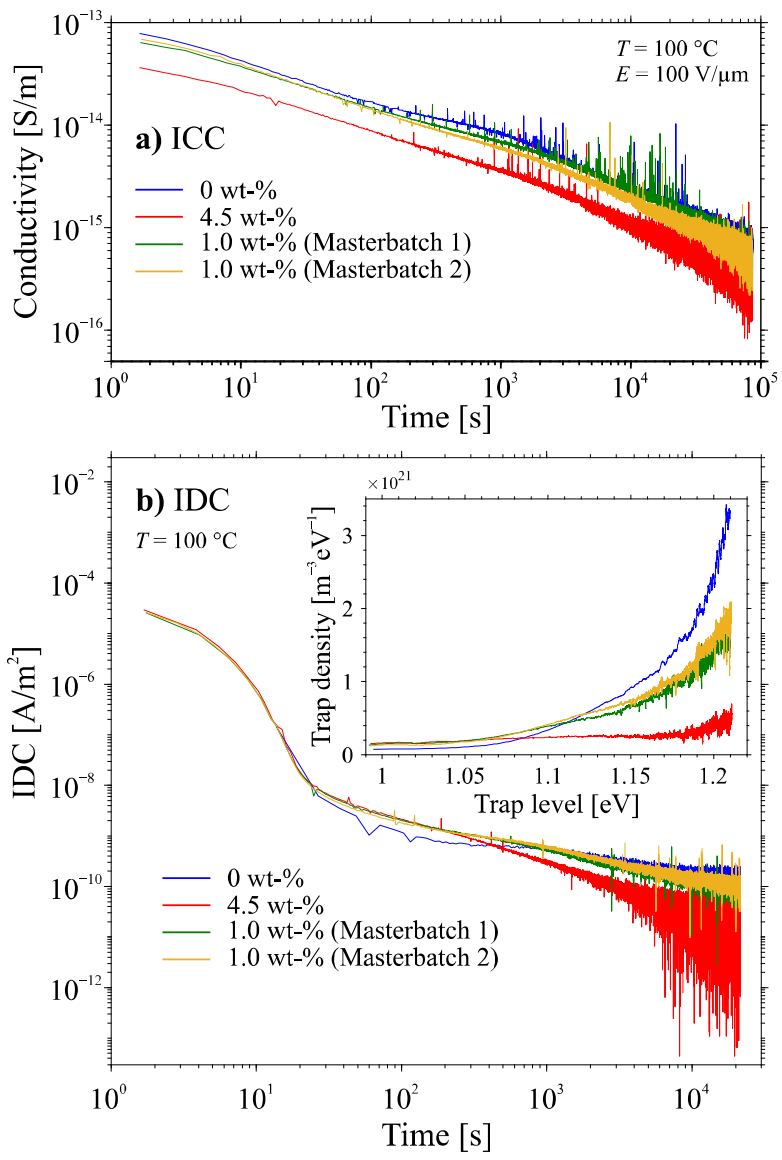

Fig. 5. Isothermal a) charging current at $100{ }^{\circ} \mathrm{C}, 100 \mathrm{~V} / \mu \mathrm{m}$ (expressed as volumetric conductivity) and b) discharge current at $100{ }^{\circ} \mathrm{C}$ of the biaxially stretched films. The inset in b) shows the trap level vs. density distribution derived from the IDC characteristics (from $\sim 20 \mathrm{~s}$ to $6 \mathrm{~h}$ ).

\section{CONCLUSIONS}

Inter-laboratory measurements on laboratory-scale thick cast films and biaxially stretched thin silica-PP films consistently indicated that incorporation of nanosilica reduces the concentration of deep traps $(\sim 1 \mathrm{eV})$ and modifies the shallow trap depth/density distribution in comparison to neat PP. This was related to (i) reduced space charge accumulation and faster depolarization characteristics in thick cast films and (ii) reduced conductivity and high-temperature dielectric loss in the silicaBOPP thin films. Such dielectric property modifications can potentially lead to benefits for insulation reliability and lifetime, especially at higher temperatures. These outcomes are not significantly affected by use of the masterbatch route, with this being advantageous as it naturally tends to minimize impurities and thermal degradation during processing and reduces the amount of nanocompound required for thin film manufacturing. For the laboratory-scale films, detailed assessment of the role of impurities and structural inhomogeneities has to be made in future work. Furthermore, measurements at higher DC electric fields and over longer time periods are seen necessary.

\section{ACKNOWLEDGMENT}

This project has received funding from the European Union's Horizon 2020 research and innovation programme under grant agreement No 720858.

\section{REFERENCES}

[1] S. K. Kumar, B. C. Benicewicz, R. A. Vaia, and K. I. Winey, " $50^{\text {th }}$ Anniversary Perspective: Are Polymer Nanocomposites Practical for Applications?," Macromolecules, vol. 50, no. 3, pp. 714-731, 2017.

[2] CIGRE Working Group D1.40, "Functional nanomaterials for electric power," 2016.

[3] I. Rytöluoto, K. Lahti, M. Karttunen, M. Koponen, S. Virtanen, and M. Pettersson, "Large-area dielectric breakdown performance of polymer films - Part II: Interdependence of filler content, processing and breakdown performance in polypropylene-silica nanocomposites," IEEE Trans. Dielectr. Electr. Insul., vol. 22, no. 4, pp. 2196-2206, 2015.

[4] M. Takala et al., "Dielectric properties and partial discharge endurance of polypropylene-silica nanocomposite," IEEE Trans. Dielectr. Electr. Insul., vol. 17, no. 4, pp. 1259-1267, 2010.

[5] G. C. Montanari, P. Seri, M. Ritamäki, K. Lahti, I. Rytöluoto, and M. Paajanen, "Performance of Nanoparticles in the Electrical Behavior of DC Capacitor Films," in 12th Int. Conf. Prop. App. Dielectr. Mat., 2018.

[6] M. Ritamäki, I. Rytöluoto, K. Lahti, T. Vestberg, S. Pasanen, and T. Flyktman, "Large-area approach to evaluate DC electro-thermal ageing behavior of BOPP thin films for capacitor insulation systems," IEEE Trans. Dielectr. Electr. Insul., vol. 24, no. 2, 2017.

[7] D. Fabiani, G. Montanari, and L. Dissado, "Measuring a possible HVDC insulation killer: Fast charge pulses," IEEE Trans. Dielectr. Electr. Insul., vol. 22, no. 1, pp. 45-51, 2015.

[8] Yewen Zhang, J. Lewiner, C. Alquie, and N. Hampton, "Evidence of strong correlation between space-charge buildup and breakdown in cable insulation," IEEE Trans. Dielectr. Electr. Insul., vol. 3, no. 6, pp. 778783, 1996.

[9] H. Li et al., "Study on the impact of space charge on the lifetime of pulsed capacitors," IEEE Trans. Dielectr. Electr. Insul., vol. 24, no. 3, pp. 1870 $1877,2017$.

[10] T. D. Huan et al., "Advanced polymeric dielectrics for high energy density applications,” Prog. Mater. Sci., vol. 83, no. 1, pp. 236-269, 2016.

[11] Q. Chen, Y. Shen, S. Zhang, and Q. M. Zhang, "Polymer-Based Dielectrics with High Energy Storage Density," Annu. Rev. Mater. Res., vol. 45, no. 1, pp. 433-458, 2015.

[12] I. Rytöluoto, M. Ritamäki, K. Lahti, S. Pasanen, and J. Pelto, "Dielectric breakdown properties of mechanically recycled $\mathrm{SiO}_{2}-\mathrm{BOPP}$ nanocomposites," in 2016 IEEE Int. Conf. Dielectr., 2016, pp. 288-292.

[13] I. Rytöluoto, A. Gitsas, S. Pasanen, and K. Lahti, "Effect of film structure and morphology on the dielectric breakdown characteristics of cast and biaxially oriented polypropylene films," Eur. Polym. J., vol. 95, 2017.

[14] G. Mazzanti, G. C. Montanari, and J. M. Alison, "A space-charge based method for the estimation of apparent mobility and trap depth as markers for insulation degradation-theoretical basis and experimental validation," IEEE Trans. Dielectr. Electr. Insul., vol. 10, no. 2, pp. 187-197, 2003.

[15] I. Rytöluoto, M. Ritamäki, and K. Lahti, "Short-Term Dielectric Performance Assessment of BOPP Capacitor Films: A Baseline Study," in 12th Int. Conf. Prop. App. Dielectr. Mat., 2018.

[16] A. Kahouli, O. Gallot-Lavallée, P. Rain, O. Lesaint, C. Guillermin, and J.-M. Lupin, "Dielectric features of two grades of bi-oriented isotactic polypropylene," J. Appl. Polym. Sci., vol. 132, no. 28, p. 42224, 2015.

[17] I. Rytöluoto, M. Ritamäki, A. Gitsas, S. Pasanen, and K. Lahti, "Morphology Development, Structure and Dielectric Properties of Biaxially Oriented Polypropylene," Proc. Nord. Insul. Symp., 2017.

[18] F. Tian, W. Bu, L. Shi, C. Yang, Y. Wang, and Q. Lei, "Theory of modified thermally stimulated current and direct determination of trap level distribution," J. Electrostat., vol. 69, no. 1, pp. 7-10, 2011.

[19] P. K. Khare and S. K. Jain, "Anomalous thermally stimulated currents and space charge in poly(vinyl pyrrolidone)," Polym. Int., vol. 49, no. 3, pp. 265-268, 2000.

[20] H. Ghorbani et al., "Long-term conductivity decrease of polyethylene and polypropylene insulation materials," IEEE Trans. Dielectr. Electr. Insul., vol. 24, no. 3, pp. 1485-1493, 2017.

[21] J. G. Simmons and M. C. Tam, "Theory of Isothermal Currents and the Direct Determination of Trap Parameters in Semiconductors and Insulators Containing Arbitrary Trap Distributions," Phys. Rev. B, vol. 7, no. 8, pp. 3706-3713, 1973.

[22] L. Qingquan, T. Fuqiang, Y. Chun, H. Lijuan, and W. Yi, "Modified isothermal discharge current theory and its application in the determination of trap level distribution in polyimide films," $J$. Electrostat., vol. 68, no. 3, pp. 243-248, 2010. 